Christian Fandrych (2011):

\title{
Wie geht es eigentlich den Halbsuffixen?
}

Diese Publikation wurde zuerst veröffentlicht / This publication has been published first in:

Deutsche Sprache 2/2011, 137 - 153. 


\title{
Christian Fandrych
}

\section{Wie geht es eigentlich den „Halbsuffixen“?}

\begin{abstract}
Der Beitrag diskutiert den Status von reihenbildenden adjektivischen Zweitgliedern, die in der Wortbildungsdiskussion der vergangenen Jahrzehnte meist einem Zwischenbereich zugeordnet wurden, der teils mit Begriffen wie „Halbaffix“ oder „Affixoid“ gefasst wurde. Es wird argumentiert, dass es sich um einen Kernbereich adjektivischer Wortbildung handelt, der mit dem Begriff der lexematischen Junktion besser gefasst werden kann, und der einen prägnanten Platz im Wortbildungssystem des Adjektivs besitzt. Dies wird im Folgenden mit einem Überblick über wichtige semantisch-funktionale Felder, in denen derartige Zweitglieder produktiv sind, überblickshaft illustriert.

This article discusses the status of productive adjectival second elements, which in the literature on wordformation in recent decades have mostly been associated with a transitional area referred to by terms such as "semi-affix" or "affixoid". The article argues that this is a core area of adjectival word-formation which is better described by the term lexematic junction, and that it has a prominent place in the word-formation system of the adjective. The article gives a survey of major semantic-functional fields in which such second elements are productive.
\end{abstract}

In den vergangenen Jahrzehnten hat in der Wortbildungsforschung die Diskussion um den Zwischenbereich zwischen Komposition und Derivation und um die Angemessenheit einer Art Zwischenkategorie (deren Elemente meist mit den Begriffen „Halbaffix“ oder „Affixoid“ bezeichnet wurden) verschiedene Wendungen genommen und war auch unterschiedlichen Konjunkturen unterworfen. Der vorliegende Beitrag nimmt insbesondere den adjektivischen Bereich in den Blick, genauer: die reihenbildenden Zweitglieder, die diesem Zwischenbereich häufiger zugeordnet werden („Halbsuffixe“/,Suffixoide“), unter anderem also etwa die folgenden Wortbildungsprodukte:

(1) arbeitsfähig, arbeitswillig, arbeitswütig, arbeitsfreudig, arbeitsgeil, arbeitsreich, arbeitsintensiv, arbeitsarm, arbeitsträchtig, arbeitsfrei, arbeitsmüde.

Dabei sollen noch einmal einige Argumente für bzw. gegen eine Halbsuffix-Kategorie gesichtet und anhand wichtiger Merkmale dieses Wortbildungsbereichs auch bewertet werden (dies ist die eine Lesart des Titels des vorliegenden Beitrags). Zum anderen aber soll aufgezeigt werden, wie produktiv dieser Wortbildungsbereich ist und wie sehr er offenbar den modernen Ausdruckbedürfnissen und -wünschen einer Gesellschaft entspricht, die so auf vergleichsweise schematische Art Benennungseinheiten (Bezugssubstantive) oder Handlungen bzw. Vorgänge nach bestimmten relevanten Eigenschaftstypen differenziert einordnen kann (das ist die zweite Lesart des Beitragstitels). Hierzu soll anhand einer Sichtung von Korpusdaten des modernen Deutsch überblickshaft dargestellt werden, welche semantisch-funktionalen Bereiche auf diese Art „erschlossen“ werden sowie die Produktivität mithilfe neuerer Belege dokumentiert werden. ${ }^{1}$

Dafür wurden das Teilkorpus „Neuakquisitionen“ von COSMAS II, das Portal „Deutscher Wortschatz“ der Universität Leipzig, das Herder-BYU-Korpus sowie auch das Internet als Quellen genutzt. Ich bin Karen Graefe (Leipzig) für die Unterstützung bei der Beleg- und Literaturrecherche zu Dank verpflichtet, Jupp Möhring danke ich für die Überprüfung von Häufigkeiten im Herder-BYU-Korpus. 


\section{Kategorielles}

Die Diskussion um die Kategorie „Affixoid/Halbaffix“ hat in den letzten Jahren wieder an Konjunktur gewonnen (vgl. etwa die Beiträge von Ascoop (2005) und Elsen (2009)). Sie stellt einen der Versuche dar, die „Unübersichtlichkeit“ in der Darstellung der Wortbildung vor allem des Adjektivs (vgl. Eichinger 2007, S. 114) zumindest mithilfe einer griffigen Benennung $\mathrm{zu}$ überwinden, ${ }^{2}$ motiviert nicht zuletzt aus dem Unbehagen einer Beschränkung der Orientierung an den Hauptkategorien Komposition und Derivation (vgl. Elsen (2009, S. 323-325) für eine Diskussion der Probleme und Inkonsistenzen, die eine solche Beschränkung teilweise mit sich bringt). ${ }^{3}$ Operiert wird meist mit einem Merkmalbündel, das sich an der alten Affixoid-Charakterisierung orientiert, wie sie etwa bei Fleischer (1982, S. 67-70) zusammenfassend dargelegt wird: Affixoide sind tendenziell gebunden, dienen der Reihenbildung und haben sich semantisch von ihrer freien lexikalischen Entsprechung zumindest in einigen Untergruppen entfernt (vgl. ausführlicher auch Kühnhold et al. 1978). In der Literatur wurde auch häufig das Merkmal der Verschiebung des Bedeutungsverhältnisses genannt (vgl. Fleischer 1982, S. 69f.), etwas, was zumindest für den adjektivischen Bereich heute systematischer als „Junktionsfunktion“" (Fandrych 1993) oder, noch allgemeiner, als ein eigenes Wortbildungsverfahren („Inkorporation“) beschrieben wird (Eichinger 2000, S. 157ff., 214ff., Eichinger 2007, S. $117 \mathrm{ff}$ ). ${ }^{4}$ Die interne Heterogenität der so gewonnenen Kategorie und die neu entstehenden Abgrenzungsprobleme werden einerseits eingeräumt, andererseits mit dem Verweis auf ähnliche Einordnungsprobleme im Randbereich von Komposition und Derivation als Gegenargument zu entkräften versucht (vgl. etwa Elsen 2009, S. 328f.).

Die Versuche einer „Wiederbelebung“ der Affixoid-Kategorie lösen eine Phase ab, in der die meisten Überblicks- und Einführungsdarstellungen eine begriffliche Fassung ganz vermieden haben oder aber eine weit differenziertere Beschreibung des so genannten Übergangsbereichs vorgenommen haben, die sich begrifflich bisher noch nicht auf breiter Basis durchgesetzt hat. ${ }^{5}$ Dabei wurde die Existenz reihenhaft ausgebauter Erst- und Zweitglieder selbst selten in Frage gestellt - die zentrale Frage war vielmehr, wie einheitlich dieser Wortbildungsbereich zu fassen ist, als wie selbstständig er im Vergleich zur Komposition und Derivation zu charakterisieren ist und wie sinnvoll daher eine begriffliche Kategorienbildung bzw. eine einheitliche summarische Bezeichnung erscheint. Sollen Erstglieder („Präfixoide“), deren Ausgangsbedeutung zugunsten einer augmentativen, evaluativen und taxativen Bedeutung (vgl. Ascoop 2005, S. 18) verblasst oder ganz verschwindet, mit schemaorientierten, semantisch häufig deutlich vom Ausgangswort ableitbaren oder auf bestimmte Lexeme beziehbaren relationalen Zweitgliedern einer Sammel-

2 Dieses Motiv legt schon der Titel von Elsen (2009) nahe.

3 Der diachrone Aspekt - auch unter dem Begriff der Grammatikalisierung gefasst (vgl. Munske 2002) spielt in der Argumentation ebenfalls immer wieder eine Rolle. Zwar ist unstrittig, dass viele gegenwärtige Suffixe durch Grammatikalisierung ehemals freier Lexeme entstanden sind, jedoch ist dies keine zwangsläufige Entwicklung und kann für eine synchrone Analyse kein entscheidendes Kriterium sein.

4 Barz (2009, S. 747ff.) stellt die Mehrzahl der hier interessierenden Bildungen zur Komposition, wo sie, ähnlich wie bei Eichinger (2000), als Rektionskomposita beschrieben werden.

5 Die Mehrzahl der neueren Überblicksdarstellungen fasst die Kategorie „Kompositionsglied“ weiter als noch Kühnhold et al. (1978) oder auch Fleischer (1982), so Motsch (2004), Fleischer/Barz (2007), Barz (2009), Donalies (2005), Lohde (2006), der Tendenz nach auch Erben (2006) und Eisenberg (2006). Den Affixoid-/Halbaffixbegriff verwenden in den Überblicksdarstellungen vor allem noch Wellmann (1995) und Naumann (2000). 
kategorie zugeordnet bzw. auch nur aus praktischen Gründen einheitlich bezeichnet werden? ${ }^{6}$ Oder ist insgesamt differenzierter zu beschreiben, mit welchen Wortbildungsprozessen wir es im Einzelfall zu tun haben - was auch für viele Phänomene gilt, die traditionellerweise unproblematisch gesehen wurden, wie dies Eichinger (2007) für die adjektivische Wortbildung vorschlägt?

Mit den folgenden Überlegungen plädiere ich für eine differenziertere und begrifflich andere Fassung dieses Wortbildungsbereichs, wobei ich insbesondere drei Argumente für zentral halte.

\subsection{Kern und Peripherie}

Bezogen auf das hier interessierende Feld des reihenhaften Ausbaus des Adjektivbestands mit relationalen Zweitgliedern lässt sich zunächst feststellen, dass es sich nicht um einen Randbereich, sondern um einen Kernbereich der adjektivischen Wortbildung handelt, was mit den Wortarteigenschaften des Adjektivs begründet werden kann (etwa der Funktion, implizite Prädikationen herzustellen). Charakteristisch ist, dass für die Reihenbildung häufig (aber nicht immer) einfache, mit Argumentstellen versehene („relationale“) Adjektive genutzt werden. Es handelt sich also typischerweise nicht um eine „Verblassung“ oder Desemantisierung von ursprünglich als Kompositionsglieder zu beschreibenden Lexemen, ${ }^{7}$ sondern die Reihenbildung setzt bei Zweitgliedern an (oder schafft diese neu), die gerade die Herstellung eines semantisch recht deutlich geprägten Bezugs zwischen Erstglied und Bezugssubstantiv (oder eine Bezugnahme auf Handlungen oder Vorgänge) erlauben. Die Existenz von freien (häufig relationalen) Varianten oder die Beziehbarkeit auf semantisch eng verwandte Wortstämme (wie bei enthalten/-haltig oder Freude/-freudig) stützt die im Vergleich zu typischen Suffixen - recht hohe semantische Festigkeit und Eindeutigkeit vieler derartiger Zweitglieder. Im Vergleich zur Transpositionsfunktion von Suffixen erlauben die Zweitglieder des oben genannten Typs eine stärkere Differenzierung bestimmter kommunikativ relevanter Eigenschaften auf schematische Art (wie etwa im Falle des obengenannten Beispiels: verschiedene Formen, in denen das Konzept Arbeit für ein Bezugssubstantiv relevant sein kann). Wie in Abschnitt 2 gezeigt wird, fächert sich dieses Wortbildungsmodell in der Gegenwartssprache in immer weitere semantische Bereiche auf und ist somit äußerst produktiv.

\subsection{Varianten der Nutzung von Relationalität}

In mancher Hinsicht vergleichbar ist ein weiterer Wortbildungsprozess, mithilfe dessen noch spezifischere Ausdifferenzierungen ermöglicht werden. Gemeint sind Bildungen mit partizipialen adjektivischen Zweitgliedern, also etwa:

(2) linguistikgeschädigt, verkehrsberuhigt, erfolgsorientiert, meerumschlungen, erdverwachsen

Solche Bildungen nutzen eine andere Art der Relationalität, nämlich diejenige, die durch

6 Interessant ist, dass sowohl Ascoop (2005) als auch Elsen (2009), die für die (Wieder-)Verwendung des Begriffs „Affixoid“ argumentieren, dies eher aus praktischen Gründen tun und damit offenbar keinen systematisch-kategoriellen Anspruch verbinden, vgl. etwa Ascoop (2005, S. 26).

7 Diese Sicht vertritt Elsen (2009, S. 326) und versucht damit, die weniger große Produktivität der, ,Suffixoide“ gegenüber den „Präfixoiden“ zu erklären. Wie im Verlaufe des vorliegenden Beitrags noch deutlicher werden wird, scheint die Reihenbildung mithilfe relationaler adjektivischer Zweitglieder aber durchaus hochproduktiv zu sein und auch auf immer weitere Abstufungsbereiche auszugreifen. 
die verbale Rektion gestiftet wird (Eichinger zählt sie zu den Rektionskomposita, vgl. Eichinger (2007, S. 117f.)). Auch sie dienen in erster Linie dazu, eine - lexikalisch noch etwas deutlichere - Beziehung zwischen einer im Erstglied genannten Größe und einem Bezugssubstantiv herzustellen - und in vielen Fällen kann das Zweitglied die Bildung ebenfalls nicht als Ganze vertreten: Während man vielleicht von einem linguistikgeplagten Studenten noch sagen kann, er sei ein geplagter Student, ist dies etwa bei verkehrsberuhigter Stadtteil, erfolgsorientierter Institutsdirektor oder auch meerumschlungenes bzw. erdverwachsenes Bundesland nicht in gleicher Weise mehr möglich. Das Zentrale an dieser Bildungsweise ist gerade die Ausnutzung der inhärenten Relationalität für die verdichtende Bildung impliziter Prädikationen. Gleichzeitig scheint doch bei der Mehrzahl solcher Bildungen keine Desemantisierung oder semantische Verblassung des Zweitglieds aufzutreten. Sie stellen ganz einfach einen weiteren Kernbereich der adjektivischen Wortbildung dar, der allerdings in besonderer Nähe zur Syntax steht.

Eine Kategorie „Affixoid“ liefert insgesamt für diese beiden Kernbereiche insofern keine Lösung, als sie - soweit jedenfalls die Kategorie bisher zu bestimmen versucht wurde die partizipialen, ,inkorporierenden“ Bildungen der traditionellen Komposition zuschlagen würde, den anderen, ebenfalls auf (allerdings lexikalischer) Relationalität basierenden Kernbereich adjektivischer Wortbildung aber davon abtrennen und als Zwischenkategorie (-oid) gesondert fassen würde. Darin kann ich keinen Benennungs- und Beschreibungsvorteil erkennen.

\subsection{Morphologische Uneinheitlichkeit und funktionale Einheitlichkeit}

Was die Sortierung im Bereich der hier im Mittelpunkt stehenden adjektivischen Zweitglieder vergleichsweise schwierig macht, ist ihre strukturelle bzw. morphologische Uneinheitlichkeit: In der Mehrheit sind die Zweitglieder auf auch frei vorkommende Adjektive mit Argumentstelle zurückzuführen. Diese Argumentstelle wird nun auf Wortbildungsebene genutzt. Das ist etwa der Fall bei -frei, -arm, -reich ebenso wie bei -feindlich, -freundlich, -fähig, -bereit, -ähnlich, -hungrig etc. Solche Bildungen lassen sich dann auch mit dem Begriff der „Inkorporation“, wie Eichinger dies vorschlägt, erklären. Einige funktional vergleichbare Zweitglieder sind aber anders entstanden, etwa durch morphologische Reanalyse von desubstantivischen Ableitungen und Verselbstständigung der neu gewonnenen Zweitglieder (-artig, -förmig, -pflichtig, etwa flaschenförmig < Flaschenform, aber nicht monstranzförmig $<*$ Monstranzform), teils auch unter Bezug auf verbale Stämme (-haltig). leer hat synchron keine Argumentstelle, so dass hier eine Argumenterweiterung geschaffen wurde (ähnlich auch etwa -intensiv, -aktiv, -intern, -extern). Diese Zweitglieder lassen sich also nicht auf einfache Weise durch das Prinzip der Inkorporation erklären, sondern nur unter der Annahme, dass hier mit morphologischen Mitteln ein den inkorporierenden Zweitgliedern vergleichbares Pendant geschaffen wurde, das sich auch sonst parallel verhält (einfache, aber klare semantische Konturierung, Zusammenspiel mit anderen reihenbildenden Zweitgliedern). Aus diesem Grund halte ich den Begriff lexematische Junktionsbildung für geeigneter. ${ }^{8}$

Eichinger (2007, S. 119) spricht auch von „lexematischen Reihen“. Der Begriff der „Junktion“ soll die spezifische relationierende Kraft zum Ausdruck bringen, die solchen Zweitgliedern inhärent ist, „lexematisch“ drückt aus, dass ein Bezug zu einem Wortstamm vorhanden ist. 
Die Grenze zwischen solchen Junktions-Zweitgliedern und dem eigentlichen Affixbereich läge dann dort, wo die Zweitglieder statt funktional-semantischer Einheitlichkeit eine (affixtypische) Multifunktionalität aufweisen und der semantische Bezug auf frei vorkommende Lexeme nicht mehr herstellbar ist. Dies ist etwa der Fall bei -mäßig, das auch in der Wortbildungsliteratur häufig bereits als Suffix betrachtet wird. ${ }^{9}$ Wie auch immer die etymologische Herleitung von mäßig zu beurteilen ist, es ist in keiner seiner drei Hauptbedeutungen als Wortbildungselement mehr auf das freie Adjektiv -mäßig zu beziehen; allerdings lässt sich in einer Untergruppe ein gewisser Zusammenhang mit dem Substantiv $M a \beta$ herstellen, etwa bei den Vergleichsbildungen: Etwas rambomäßig machen etwa wäre zu lesen im Sinne von, etwas in ebensolcher Weise / im selben (gewalttägigen) Maße erledigen wie Rambo dies üblicherweise tut'. In anderen Verwendungen ist mäßig synonym zu -gemäß (vorschriftsmäßig), während im allgemeinsten Fall -mäßig der syntaktisch flexiblen Verfügbarmachung von nominalen Inhalten dient. Hier wird es häufig auch zur Topikalisierung genutzt, die Bedeutung könnte mit ,Geltungsbereich festlegen' umschrieben werden, vgl.:

(3) Man guckt gespannt und wartet und wartet und denkt, na ja, gesagt wird ja schon, dass es in Braunschweig spielt, aber was man sieht, Straßen, Bungalows, 'ne Bäckerei, ... ein paar Straßen, das ist kaum typisch braunschweigisch, das könnte überall sein. ... Stadtmarketingmäßig ist der Tatort mit dem schönen Titel „Es wird Trauer sein und Schmerz“ also eher eine Null-Nummer - bis dahin. (www.newsclick.de/23.10.2009)

Hier wird der Unterschied zu semantisch doch klarer instruierenden Junktions-Zweitgliedern wie -frei, -arm; -hungrig, -trächtig; -pflichtig, -förmig deutlich.

Vielleicht sollte insgesamt stärker die Funktionalität bestimmter Bildungsbereiche im Vordergrund der Klassifikation stehen: Bei den hier als adjektivische Junktionsbildungen bezeichneten Zweitgliedern handelt es sich letztlich um ein an kommunikativen Bedürfnissen orientiertes, produktorientiertes Muster, das mit unterschiedlichen Mitteln im Ergebnis zu ähnlich abstrakt klassifizierenden Junktoren führt. Hierfür werden, wo vorhanden, relationale adjektivische Zweitglieder genutzt; wo diese nicht vorhanden sind, werden über verschiedene morphologische Verfahren unter Rückgriff auf geeignete Wortstämme Zweitglieder hergestellt, deren semantische Funktion auf diese Weise intuitiv vergleichsweise eindeutig ist (-förmig, -artig, -haltig; mit Abstrichen, da meist noch als desubstantivisches Derivat interpretierbar auch bei -pflichtig). Daneben sind mindestens noch einige Fälle anzusetzen, bei denen die Argumentstelle erst geschaffen wird bzw. in der freien Form nicht angelegt ist; dies ist etwa der Fall bei leer, ähnlich auch intensiv (pflegeintensiv), aktiv (wortbildungsaktiv), intern und extern in Bildungen wie IDS-intern und parlamentsextern.

\section{Domänen der lexematischen Junktionsbildung (,Halbaffixe“)}

Im Folgenden soll versucht werden, die semantischen Domänen der reihenbildenden adjektivischen Zweitglieder überblicksartig darzustellen, um einen etwas systematischeren Eindruck von der Funktionsbreite dieses Wortbildungsbereichs zu geben. Die Übersicht kann keinen Anspruch auf Vollständigkeit besitzen. Ich stütze mich im Wesentlichen auf die Übersichten bei Wellmann (1995), Weinrich (2003), Eichinger (2000), Motsch (2004),

9 Obwohl auch hier keine Einheitlichkeit herrscht: So stufen Altmann/Kemmerling (2005) das Morphem „formal als Determinativkompositum“, semantisch aber eher als Halbsuffix ein. Auch Eisenberg (2006) sieht -mäßig wie auch -frei als Halbaffixe. 
Lohde (2006) und Barz (2009). Wir befinden uns dabei auch in der Tradition der umfassenden Bände zur Wortbildung des IDS (insbesondere Kühnhold et al. 1978). Es ist allerdings, so denke ich, an der Zeit, einen Versuch zu unternehmen, die reihenbildenden Zweitglieder umfassend nach semantisch-funktionalen Gruppen zu ordnen und auch ihr Zusammenspiel mit Suffixen einerseits, mit anderen Mitteln der adjektivischen Wortbildung (Präfigierung, Bildungen mit Partizipien als Zweitgliedern) andererseits genauer und systematischer zu untersuchen. Dabei sollten auch ihre jeweiligen Kommunikationsdomänen (fachliche/administrative/umgangs-/alltagssprachliche Zusammenhänge etc.) sowie die Produktivitätsdynamik mit berücksichtigt werden. Dies kann an dieser Stelle nicht umfassend erfolgen. Ziel ist vielmehr, v.a. die Breite des semantisch-funktionalen Spektrums dieses Wortbildungsbereichs zu verdeutlichen, aufzuzeigen, welch ausdifferenzierte Schematisierungen auf diese Weise ermöglicht werden und exemplarisch Produktivitätsrichtungen und Tendenzen der allgemeinen Häufigkeit anzudeuten. ${ }^{10}$

\subsection{Semantisch-funktionale Gruppen und Ausdifferenzierung}

Schon mehrfach ist festgestellt worden, dass reihenbildende Zweitglieder (lexematische Junktoren) dazu dienen, Handlungen modalisierend als Eigenschaften verfügbar zu machen. In Anlehnung an Barz (2009, S. 754ff.) kann man hier zwischen aktivisch-modalen und passivisch-modalen Handlungseinordnungen unterscheiden.

\section{a) Aktivisch-modal: Kernbedeutung: Y kann X machen / x-en}

Die folgende Übersicht zeigt zentrale Suffixe und lexematische Junktoren (gebundene Zweitglieder ebenso wie solche, die auch frei vorkommen), die das aktivisch-modale Feld ausdifferenzieren: ${ }^{11}$

\begin{tabular}{|c|c|c|}
\hline Suffixe & $\begin{array}{l}\text { gebundene Zweit- } \\
\text { glieder }\end{array}$ & Zweitglieder \\
\hline \multicolumn{3}{|c|}{ Y kann X machen / X-en } \\
\hline -bar (schwimmbar, brennbar) & & fähig (arbeitsfähig, tauchfähig) \\
\hline \multicolumn{3}{|l|}{-(er)isch (misstrauisch) } \\
\hline \multicolumn{3}{|l|}{-(er)lich (befremdlich) } \\
\hline \multicolumn{3}{|l|}{-ig (feinfühlig) } \\
\hline \multicolumn{3}{|l|}{-un-...lich (unaufhörlich) } \\
\hline \multicolumn{3}{|c|}{ Y macht X oft / gerne / intensiv / effektiv / schnell } \\
\hline -ig (zappelig) & \begin{tabular}{|l|} 
freudig (drehfreudig, \\
wuchsfreudiger Baum, \\
zweikampffreudiger \\
Spieler) \\
\end{tabular} & lustig (kauflustig, kampfeslustig) \\
\hline -haft (naschhaft) & & aktiv (stoffwechselaktive Bakterien) \\
\hline -sam (arbeitsam) & & froh (lebensfroh, arbeitsfroh) \\
\hline \multicolumn{3}{|c|}{ Y hat (generell) eine große / zu große Disposition dazu, $\mathrm{X}$ zu machen } \\
\hline & & willig (leistungswillig, steuerwillig) \\
\hline
\end{tabular}

$\overline{10}$ Letzteres erfolgt hier vor allem durch eine - nicht systematische - Korpusabfrage, v.a. von COSMAS II sowie des Wortschatz-Portals der Universität Leizpzig.

11 Fettdruck signalisiert besondere Produktivität bei den lexematischen Junktionsbildungen. Bemerkungen zur Basis und zur Produktivität finden sich stichpunktartig uner der Übersicht. 


\begin{tabular}{|l|l|l|}
\hline & & hungrig (spielhungrig, reisehungrig) \\
\hline & & süchtig (kinosüchtig, fernsehsüchtig) \\
\hline & & verrückt (reiseverrückt) \\
\hline & wütig (arbeitswütig, tratschwütig) \\
\hline & geil (fernsehgeil, arbeitsgeil) \\
\hline \multicolumn{3}{|c|}{ Y hat aktuell die Disposition dazu, X zu machen } \\
\hline \multicolumn{3}{|c|}{ Y kann X besonders gut machen } \\
\hline & bereit (abflugbereit, einsatzbereit) \\
\hline Y hat wenig Disposition dazu, X zu machen \\
\hline \\
\hline
\end{tabular}

\begin{tabular}{|l|l|l|}
\hline $\begin{array}{l}\text { Basis: Verb, bei -ig auch } \\
\text { Verbgruppe }\end{array}$ & $\begin{array}{l}\text { Basis: verbal, teils } \\
\text { substantivisch }\end{array}$ & $\begin{array}{l}\text { Basis: meist substantivisch, teils auch verbal, } \\
\text { häufig deverbal }\end{array}$ \\
\hline $\begin{array}{l}\text { Insgesamt wenig produktiv } \\
\text { (vgl. Eichinger 2000, S. 154; } \\
\text { Motsch 2004, S. 297) }\end{array}$ & & $\begin{array}{l}\text { Unterschiedlich produktiv, einige hochpro- } \\
\text { duktive Gruppen (fett gedruckt) }\end{array}$ \\
\hline
\end{tabular}

Tabelle 1: aktivisch-modaler Bereich

Es wird deutlich, dass gerade im aktivisch-modalen Bereich, wo die Derivationstypen insgesamt nur schwach entfaltet und kaum produktiv sind (vgl. Eichinger 2000, S. 154), die reihenbildenden Zweitglieder eine echte kommunikative Lücke füllen. Die Belegauswahl zeigt, dass die kommunikativen Domänen dabei recht unterschiedlich sein können: vom ungeheuer produktiven und allgegenwärtigen fähig über stärker Fachlichkeit oder doch Sachlichkeit signalisierende Zweitglieder wie -freudig, willig, bereit, faul, müde bis hin zu alltagssprachlichen oder gar stark umgangssprachlich markierten Bildungen (verrückt, vgl. reiseverrückt; wütig, vgl. arbeitswütig; v.a. geil, vgl. fernsehgeil und arbeitsgeil). Wo Bildungsmuster Fach- bzw. Sachlichkeit markieren, eignen sie sich besonders gut für eine „uneigentliche“, diese Markierung konterkarierende Verwendung. Dies zeigt sich etwa in den Belegen brunftlustig und lachwillig. In solchen Kontexten haftet diesen Reihenbildungen etwas Umständliches und übermäßig Schematisierendes an, das offenbar alltagssprachlichen Stilvorstellungen nicht - oder nur ironisierend - entspricht: ${ }^{12}$

(4) Ich weiss, dass ich tatsächlich nur bis zu einem gewissen Grad multitasking-fähig bin, während die meisten Frauen, die ich kenne, wirklich besser organisieren können - das muss ich neidlos anerkennen. (ngz-online.de vom 22.9.2006)

(5) Insbesondere bei der Geflügelpest ist der Viruserreger mutationsfreudig und anpassungsfähig. (COSMAS II)

(6) Dabei ist Interpols in epischer Gitarrenschraddelbreite angelegte Musik so spaßig wie eine nächtliche Krötenwanderung über eine vielbefahrene Landstraße: strebsam und brunftlustig, aber auch voll dunkler Vorahnungen, leisem Leiden und tragischer Geräusche. (welt.de

\footnotetext{
12 Hervorhebung der Belege von mir.
} 
vom 13.4.2005)

(7) Die deutsche Mittelschicht ist ein eigenartiges Wesen, eher zurückhaltend als auftrumpfend, eher depressiv als leistungsfroh. (COSMAS II)

(8) Müssten Steuerberater von Klienten leben, die Lehrer sind [...], müssten sie in Deutschland verhungern. [...] Aber Kleinvieh macht auch Mist und deshalb werden Akten von Lehrern sicher in der Frühstückspause von Praktikanten bearbeitet. Doch wenn die richtigen Klienten klingeln, dann verschwinden selbst die dünnen Akten in der Ablage „Nackte Frauen. Nackte Männer. Beide steuerpflichtig und steuerwillig!“(COSMAS II)

(9) „Das ist toll“, erzählt die 55-jährige Ingrid Thomsen, die im Lachclub Flensburg/Nordfriesland einmal monatlich etwa 20 lachwillige Norddeutsche anleitet. (morgenweb.de vom 6.5.2006)

(10) Die Tischtennisabteilung der DJK Dossenheim 1927 geht spielhungrig und selbstbewusst in das neue Jahr. (COSMAS II)

(11) 3,6 Millionen reisehungrige Leser, 1 Buchung. Süddeutschlands größter Reisemarkt.

(12) Cornelia Schroeder: „Ich bin auch früher nicht besonders reiseverrüickt gewesen, aber unser neues Haus hat exotische Reisewünsche weiter abgeschwächt. (COSMAS II)

(13) Boshaft, herrschsüchtig, tratschwütig und ganz furchtbar einsam ist die. (welt.de vom 12.4.2005)

(14) Wir Deutschen werden immer arbeitsgeiler! (COSMAS II)

(15) Lieber fernsehgeil als radioaktiv. (Dieter Bohlen, mittlerweile als Klingelton bei T-Mobile herunterladbar)

(16) Türken mit Hauptschulabschluss sind wesentlich weniger integrationsbereit als junge Türken mit Abitur, berichtet das Magazin „Focus“ aus einer noch unveröffentlichten Studie der Universität Bonn. (frankenpost.de vom 14.11.2005)

(17) Seit Sokrates die immer selben Sprüche: luxusorientiert, bildungsfaul, oberflächlich, arbeitsscheu. (berlinonline.de vom 15.1.2006)

Systematisch zu überprüfen wäre, wie sehr sich bestimmte Muster verselbstständigt haben bzw. wie sehr sie (noch) auf entsprechende Substantive bezogen werden können (Kauflust, Kampfeswille, Drehfreude). Weiter ist teilweise Antonymbildung mit un- möglich, dies scheint ein Hinweis auf semantische Nähe zur freien Verwendung zu sein, vgl. kampfunfähig, dialogunfähig; zeugungsunlustig, therapieunwillig (Belege aus dem Portal Deutscher Wortschatz). Deutlich ist, dass keine Scheu vor Hybridbildungen besteht (vgl. Beleg 4).

\section{b) Passivisch-modal; Kernbedeutung: Y kann ge-x-t werden}

Ein zweites semantisch-lexikalisches Feld, in dem lexematische Junktionsbildungen äußerst produktiv sind und ein Gegenstück zum ebenfalls stark ausgebauten Suffix-System darstellen, sind die Bildungen mit passivisch-modaler Bedeutung. Auch hier finden sich weit mehr Zweitglieder, als man gemeinhin in Überblicksdarstellungen findet, und die semantische Ausdifferenzierung ist beträchtlich, vgl.: 


\begin{tabular}{|c|c|c|}
\hline Suffixe & gebundene Zweitglieder & Zweitglieder \\
\hline \multicolumn{3}{|c|}{ Y kann ge-x-t werden } \\
\hline -bar (aushandelbar) & & fähig (biegefähig, zitierfähig) \\
\hline \multicolumn{3}{|l|}{-lich (begreiflich) } \\
\hline \multicolumn{3}{|l|}{-sam (biegsam) } \\
\hline \multicolumn{3}{|l|}{$\begin{array}{l}\text {-un-...lich /-bar /-sam } \\
\text { (unglaublich, unverkennbar, } \\
\text { unaufhaltsam) }\end{array}$} \\
\hline \multicolumn{3}{|c|}{ Y sollte / muss (viel) ge-x-t werden } \\
\hline & -pflichtig (impfpflichtig) & wert (lesenswert, beachtenswert) \\
\hline & & würdig (förder-, diskussionswürdig) \\
\hline \multicolumn{3}{|c|}{ Y muss immer wieder / aktuell ge-x-t werden } \\
\hline & & intensiv (arbeits-, pflegeintensiv) \\
\hline & & anfällig (reparaturanfällig) \\
\hline & & bedürftig (hilfsbedürftig, reformbedürftig) \\
\hline \multicolumn{3}{|c|}{ Y kann jederzeit / bei Bedarf ge-x-t werden } \\
\hline & & fertig (bezugsfertig, versandfertig) \\
\hline & & bereit (verzehrbereit) \\
\hline \multicolumn{3}{|c|}{ Y muss nicht / nur wenig ge-x-t werden / braucht kein / wenig X } \\
\hline \multirow[t]{2}{*}{-los (bargeldlos) } & & frei (pflegefrei, wartungsfrei) \\
\hline & & arm (pflegearm, wartungsarm) \\
\hline $\begin{array}{l}\text { Basis = Verb/Substantiv einer } \\
\text { komplexeren Prädikation }\end{array}$ & & $\begin{array}{l}\text { Basis = Verb, auch nominalisierte Infinitive } \\
\text { oder Substantive, die Teil von komplexeren } \\
\text { Prädikaten sind (etwa - anfällig) }\end{array}$ \\
\hline $\begin{array}{l}\text {-bar ist hochproduktiv, -los in } \\
\text { dieser Funktion nicht }\end{array}$ & & Teils hochproduktiv (fett gedruckt) \\
\hline
\end{tabular}

Tabelle 2: semantisch-lexikalischer Bereich I

Zudem entsteht im Bereich der Zweitglieder teils ein antonymisches System:

\begin{tabular}{|l|l|}
\hline kann / muss / sollte ge-x-t werden & \multicolumn{1}{c|}{ vs. muss nicht /wenig ge-x-t werden } \\
\hline pflegeintensiv, wartungsintensiv/-bedürftig/ & frei (pflegefrei, wartungsfrei) \\
\cline { 2 - 3 } -anfällig ... & arm (pflegearm, wartungsarm) \\
\hline steuerpflichtig, beitragspflichtig & steuerfrei, beitragsfrei \\
\hline
\end{tabular}

Tabelle 3: semantisch-lexikalischer Bereich II

-verdrossen, das bei Weinrich (2003, S. 1007) auch genannt wird, ist nicht produktiv und wenig frequent.

Gleichzeitig verbinden sich bestimmte implizite Bewertungen und Stilkonnotationen mit vielen Zweitgliedern, die sich teils wohl auch auf die Semantik ihrer freien Entsprechungen zurückführen lassen: Während -bedürftig, -anfällig, meist wohl auch -intensiv negativ konnotierte Eigenschaften benennen, sind Bildungen mit -wert, -würdig, -fertig, -bereit und -frei positiv bewertet. Fachlichkeit signalisieren wohl vor allem Bildungen auf 
-pflichtig, -fähig, -fertig. Einige Zweitglieder sind schon länger im Umlauf und darum unauffälliger Bestandteil der Gemeinsprache (etwa würdig, wert). Positive/negative Konnotationen sowie Fachlichkeitssignale sind ausschließlich auf die adjektivischen Junktionsbildungen beschränkt, die hier vorkommenden Suffixe sind in dieser Hinsicht neutral.

Auch hier sollen einige Beispiele die Produktivität dieser Zweitglieder belegen:

(18) Er hielt Frau und Tochter nicht für abschiebefähig, weil die Mutter „schon unter mitteleuropäischen Bedingungen“ nicht mehr in der Lage gewesen sei, sich um das Kind zu kümmern. (fr-aktuell.de vom 22.6.2005)

(19) Über Dinge die N macht, die N mag, die N nicht mag, die doof, wtf- oder lol-würdig sind, die schön sind oder die süchtig machen. (Beleg Chatforum) ${ }^{13}$

(20) Was ist daran jetzt ungewöhnlich und meldewert? (focus.msn.de vom 22.9.2006)

(21) Patienten, die gefüttert werden müßten und besonders pflegeintensiv seien, gerieten wegen der abgespeckten Personalsituation in den Kliniken in Schwierigkeiten. (welt.de vom 26.1.2005)

(22) Auch die traditionell Terror-anfällige Ölindustrie verdaute den Londoner Schreck schnell. (Quelle: handelsblatt.com vom 12.7.2005)

(23) Die Maren Eggert bis unter die Achseln reichende Judith Rosmair ist die eine Workshopbedürftige Frau, Maren Eggert die andere. (welt.de vom 8.1.2005)

(24) Leider ist diese sinnvolle Hilfe bei den schwächeren Versionen mit 190 und 299 PS aufpreispflichtig. (sueddeutsche.de vom 8.2.2006)

(25) Mehrere Friteusen waren gleichzeitig im Einsatz und so konnten in einer halben Stunde rund 200 halbe Hähnchen verzehrfertig aufgetischt werden. (morgenweb.de vom 8.8.2006)

(26) Doch im Gegensatz zu diesem genügt den Beutel-Drinks Wasser allein nicht, um verzehrbereit zu sein. (berlinonline.de vom 2.7.2006)

Deutlich wird auch hier die immer weiter fortschreitende Ausdifferenzierung der Modalität, die über die reihenbildenden Zweitglieder ermöglicht wird:

-pflichtig: etwas muss gemacht werden

wert und würdig: etwas verdient, gemacht zu werden (Lohde 2006, S. 159), sollte gemacht werden

fertig/bereit: Hier wird auf die Vorgeschichte einer erwartbaren Handlung Bezug genommen: etwas ist in einen Zustand/eine Situation gebracht worden, die eine Anschlusshandlung damit erlaubt.

anfällig und intensiv: es besteht eine wiederholte Notwendigkeit

bedürftig: es besteht eine akute/wiederholte Notwendigkeit

In vielen Fällen kommt es auch $\mathrm{zu}$ direkter Konkurrenz (sehenswert/-würdig, wartungsanfällig/-intensiv) mit gewissen semantisch-konnotativen Nuancen.

13 wtf ist eine gängige (jugendsprachlich-saloppe) Abkürzung für what the $f . .$, lol für laugh out loud. Beide treten v.a. in elektronischer Kommunikation auf und sind auch in deutschen Chatforen, Blogs etc. häufig. 
2.2 Haben-/Nicht-Haben: Kernbedeutung Y hat / hat kein / hat wenig X

Ein weiterer zentraler Bereich der reihenbildenden Zweitglieder ist derjenige der Possessiv-/Privativ-Relation, vgl.:

\begin{tabular}{|c|c|c|}
\hline Suffixe & gebundene Zweitglieder & Zweitglieder \\
\hline \multicolumn{3}{|c|}{ haben } \\
\hline -haft (schmackhaft) & -haltig (salzhaltig) & voll (geschmackvoll) \\
\hline -ig (staubig) & & reich (baumreich) \\
\hline -isch (neidisch) & & intensiv (klangintensiv) \\
\hline -lich (ängstlich) & & kräftig (zahlungskräftig) \\
\hline -be-/ge-...t (gestreift) & & schwanger (unheilsschwangere Musik) \\
\hline \multicolumn{3}{|c|}{ zur Konsequenz haben } \\
\hline & & trächtig (kostenträchtige Dienstleistung) \\
\hline \multicolumn{3}{|c|}{ nicht / wenig haben } \\
\hline \multirow[t]{3}{*}{-los (lustlos) } & & frei (bildschirmfreie Woche, spannungsfrei) \\
\hline & & $\operatorname{arm}($ salzarm) \\
\hline & & leer (ausdrucksleer) \\
\hline \multicolumn{3}{|c|}{ nicht / kaum zur Konsequenz haben } \\
\hline \multirow[t]{2}{*}{-los (verlustlose Lagerung) } & & frei (abgasfreie Stadtautos) \\
\hline & & arm (abfallarme Produktion) \\
\hline
\end{tabular}

\begin{tabular}{|l|l|l|}
\hline $\begin{array}{l}\text { Basis }=\text { Substantiv, bei -ig } \\
\text { auch subst. Fügung }\end{array}$ & Basis = substantivisch, teils verbal \\
\hline (bedingt) aktiv & & Produktiv, vor allem im privativen Bereich \\
\hline
\end{tabular}

Tabelle 4: Bereich der Possessiv-/Privativ-Relation

Einige dieser Zweitglieder wurden bereits mehrfach ausführlicher besprochen (vgl. etwa Fandrych 1993), ihre Produktivität ist allerdings ungebrochen. Weitere inzwischen auch recht produktive Zweitglieder dienen der Ausdifferenzierung in diesem Bereich, v.a. -intensiv (vgl. Graefe 2009), daneben -kräftig, -schwanger, -trächtig. Auch hier erfolgt der Produktivitätsschub offenbar auf der Seite der lexematischen Junktionsbildungen, weniger im Bereich der Suffixe. Inhärente Bewertungen spielen teils für die Produktivität eine wichtige Rolle (das gilt wohl v.a. für tendenziell positiv wertendes -intensiv und -frei). Einige Belege sollen auch hier die Produktivität belegen:

(27) So leidenschaftlich, so klangintensiv und farbgesättigt, so dem Vorurteil fader Harmlosigkeit entrissen hat man diese Musik wohl kaum je gehört. (archiv.tagesspiegel.de vom 12.6.2005)

(28) Dieser hellsichtige und sprachkräftige Essay gehört deshalb zu den wichtigsten Selbstreflexionen deutscher Juden; er liegt nun im Jüdischen Verlag im Suhrkamp Verlag in einer neuen Ausgabe vor. (welt.de vom 18.6.2005)

(29) Leupold ließ bei der Vorstellung ihres Beitrages wenig Raum für sehnsuchtsschwangere Impressionen. (welt.de vom 5.9.2006)

(30) Den Web-Journalismus hat OE24 auch damit nicht neu erfunden, führt im deutschsprachigen Raum aber eine Fülle recht peppiger wie klickträchtiger Präsentationsformen ein. 
(spiegel.de vom 4.9.2006)

(31) Das Endziel wäre ein springkrautfreier Regen“, sagt er und muss selbst lachen, denn „,ür solche Ziele braucht man selbst mit vielen Helfern bestimmt 15 Jahre". (pnp.de vom 13.6.2006)

(32) Heike Makatsch hat keine schlechte Stimme, doch ihre sicherlich tief empfundene, aber nuancenarme Vertonung nervt am Ende noch mehr als die filmischen Ungereimtheiten. (de.news.yahoo.com vom 22.8.2005)

Verschiedene Zweitglieder rufen unterschiedliche Konnotationen auf; die eher fachlichen -frei, -arm, -haltig, -intensiv stehen eher stilistisch gehobenen -leer, -voll, -schwanger gegenüber. Mit -los konkurriert ein Suffix, das von der Monofunktionalität und der Funktionsweise den lexematischen Zweitgliedern sehr nahe steht.

\subsection{Vergleich der Art: Y ist (bezüglich eines Merkmals) X ähnlich / an X orientiert}

Vergleiche sind ein weiterer wichtiger und produktiver Bereich für die lexematische Junktion. Auch hier werden in sich relationale Adjektive zur morphologisch-syntaktischen Verdichtung genutzt (-ähnlich, -gleich; -gemäß, -getreu, -konform), während andere Zweitglieder morphologisch - mit Bezug auf Wortstämme freier Lexeme - „hergestellt““ werden, wiederum unter Zuhilfenahme des Suffixes -ig (-artig $<$ Art; förmig $<$ Form; widrig $<$ wider).

\begin{tabular}{|c|c|c|}
\hline Suffixe & gebundene Zweitglieder & Zweitglieder \\
\hline \multicolumn{3}{|c|}{$\mathrm{Y}$, das $\mathrm{X}$ bezüglich Art / Form / Eigenschaft ähnlich ist $\mathrm{Y}$, das mit $\mathrm{X}$ identisch ist } \\
\hline -haft (katzenhaft) & -artig (torschlusspanikartig) & ähnlich (gottähnlich) \\
\hline -ig (affig) & -förmig (monstranzförmig) & gleich (kometengleich) \\
\hline \multicolumn{3}{|l|}{-isch (tyrannisch) } \\
\hline \multicolumn{3}{|l|}{-lich (menschlich) } \\
\hline \multicolumn{3}{|l|}{-mäßig (уирріетӓßig) } \\
\hline \multicolumn{3}{|c|}{$\mathrm{Y}$, das sich an $\mathrm{X}$ orientiert } \\
\hline \multirow[t]{3}{*}{-mäßig (vorschriftsmäßig) } & & gemäß (ordnungsgemä $\beta$ ) \\
\hline & & getreu (originalgetreu) \\
\hline & & konform (verfassungskonform) \\
\hline \multicolumn{3}{|c|}{$\mathrm{Y}$, das sich nicht an $\mathrm{X}$ orientiert } \\
\hline & -widrig (planwidrig) & \\
\hline
\end{tabular}

Tabelle 5: Vergleiche

Auch hier kommt es zu Antonymiebeziehungen: -widrig steht im Gegensatz zu Bildungen auf -gemäß, -getreu und -konform. -mäßig konkurriert in einer seiner Unterbedeutungen mit -ähnlich und -gleich (,wie ein X'), vgl. yuppiemäßig vs. yuppieähnlich; in einer weiteren Unterbedeutung konkurriert es mit -gemäß (vorschriftsmäßig vs. vorschriftsgemäß). Die Einordnung von -mäßig als Suffix ist sicherlich auch eine Ermessensentscheidung. Einige Belege sollen auch hier wiederum die Produktivität der Zweitglieder illustrieren:

(33) $\mathrm{Zu}$ erklären ist das nur dadurch, daß der Markt durch Unsummen verfügbaren Geldes schnurrt ..., gleichzeitig aber die Top-Qualität fehlt, und Sammler torschlußpanikartig kaufen, nach dem Motto: Lieber einem mäßigen Picasso als gar keinen. (welt.de vom 11.3.2006) 
(34) Und er beruht, das trägt er monstranzförmig vor sich her, ohne es zu müssen, auf einer wahren Begebenheit. (welt.de vom 2.3.2006)

(35) Wenn das nicht gottähnlich (zumindest halbgottähnlich) ist! (ngz-online.de vom 16.2.2006)

(36) Der sagenhafte Aus- und Aufreißer aus der Provinz avancierte kometengleich zum neidisch bewunderten „Titelrollenmann“ des Berliner Ensembles (Coriolan, Woyzeck, Danton, Baal, Puntila, Fatzer, Galilei). (welt.de vom 5.9.2005)

(37) Noch bizarrer machte den völlig ambitionsfreien Wolfsburger Vortrag der komagleiche Zustand, in dem der Gegner die Partie aufgenommen hatte. (fr-aktuell.de vom 21.3.2005)

(38) Die sandgefüllten Buddelkästen mit Werbesegel habe er nur widerwillig akzeptiert, nachdem er die ursprüngliche Idee der Stiftung Denkmalschutz abgelehnt und eine „Strandbadgemäße Gestaltung" verlangt hatte, so Baustadtrat Gröhler. (archiv.tagesspiegel.de vom 6.4.2006)

(39) Die Schau zeigt bis zum 1. November 35 maßtabsgetreue Modelle, die nach Originalzeichnungen da Vincis gebaut wurden. (swr.de vom 25.8.2006)

(40) Hoëcker: Da habe ich eine nicht studentenkonforme Meinung. (spiegel.de vom 6.8.2006)

(41) Coolness-Faktor: iPhone-mäßig hoch. (Hamburger Morgenpost, 23.6.2009)

\subsection{Affinität: Y hat große Affinität/Distanz zu X}

Was Weinrich (2003, S. 1008) als „Zweitglieder der Orientierung“ fasst, soll hier etwas konkreter unter den Begriff der ,Affinität ${ }^{`}$ gruppiert werden - im eigentlich lokalen wie im übertragenen Sinne, vgl.:

\begin{tabular}{|l|l|l|}
\hline Suffixe / Erstglieder & gebundene Zweitglieder & Zweitglieder \\
\hline \multicolumn{2}{|c|}{ Y hat große Affinität / räumliche Nähe zu X } \\
\hline -isch (parlamentarisch) / & & intern (IDS-intern) \\
\hline inner-(innerparlamentarisch) & nah (stadtnah, parteinah) \\
\hline \multicolumn{3}{|c|}{ Y hat große Distanz zu X } \\
\hline außer- (außerparlamentarisch) & & extern (parlamentsextern) \\
\hline & & fern (bildungsfern) \\
\hline & & fremd (alltagsfremd) \\
\hline
\end{tabular}

Tabelle 6: Bereich der Affinität

Auch Bildungen in diesem Bereich sind äußerst produktiv und bilden, wie die Übersicht zeigt, ein antonymisches System, vgl. auch die folgenden Belege:

(42) Frontal und rampennah positioniert er die Figuren, lässt sie emotionsfrei Glaubens- und Gewissensfragen äußern. (archiv.tagesspiegel.de vom 2.2.2005)

(43) Töpfer, noch bis Januar Leiter des Uno-Umweltsprogramms, gilt in ökologischen Fragen als grünen-nah. (spiegel.de vom 21.9.2005)

(44) Denn ehemals internetferne Bevölkerungsgruppen wie ältere Menschen und Nicht-Berufstätige fänden zunehmend den Weg ins Netz. (handelsblatt.com vom 7.9.2005)

(45) Die Werte, die stattdessen bemüht werden, insbesondere Demokratie und Rechtsstaatlichkeit, sind ebenso ehrenwert wie alltagsfremd. (fr-aktuell.de vom 7.6.2005) 
Häufig liegen institutionelle oder abstrakte Bezüge vor, bei nah und fern kann in Einzelfällen allerdings konkrete räumliche Nähe bzw. Distanz gemeint sein (randnah, stadtfern).

Insgesamt signalisieren auch diese Bildungen meist einen gewissen Fachlichkeitsgrad, wie dies auch das im öffentlichen Diskurs so frequente Beispiel bildungsfern(e Schichten) zeigt. Mit intern und extern konkurrieren die Erstglieder inner- und außer- (vgl. schon Latour 1976).

\subsection{Unversehrtheit: $\mathrm{Y}$ ist vor $\mathrm{X}$ sicher / bleibt im Kontext $\mathrm{X}$ unversehrt}

Sehr produktiv für reihenhafte adjektivische Junktionsbildung ist das semantische Feld ,Schutz/Anfälligkeit' bestimmter Bezugssubstantive gegenüber bestimmten Einwirkungen (meist von außen). Hier kommt es zur Konkurrenz einer ganzen Reihe von Zweitgliedern, die insbesondere die Beständigkeit ausdrücken, vgl.:

\begin{tabular}{|c|c|c|}
\hline Suffixe & gebundene Zweitglieder & Zweitglieder \\
\hline \multicolumn{3}{|c|}{$\mathrm{Y}$ ist vor $\mathrm{X}$ sicher } \\
\hline & & fest (hitzefest) \\
\hline & & sicher (virensicher) \\
\hline & & resistent (sonntagsredenresistent) \\
\hline & & beständig (wetterbeständig) \\
\hline & & dicht (luftdicht) \\
\hline \multicolumn{3}{|c|}{ Y bleibt im Kontext $\mathrm{X}$ unversehrt } \\
\hline & & fest (spülmaschinenfest) \\
\hline \multicolumn{3}{|c|}{$\mathrm{Y}$ ist vor $\mathrm{X}$ wenig sicher / wird von $\mathrm{X}$ stark beeinflusst } \\
\hline & & empfindlich (druckempfindlich) \\
\hline & & sensibel (konjunktursensibel) \\
\hline
\end{tabular}

Tabelle 7: Bereich ,Schutz/Anfälligkeit‘

Deutlich wird hier (wie teils auch in den anderen Gruppen), dass positive Bewertung - wo überhaupt bewertet wird - deutlich häufiger ist als negative Bewertung. Impliziert wird durch die Wortbildungsmuster, dass das Bezugssubstantiv potenziell anfällig gegenüber einer im Erstglied genannten Größe ist, diese Anfälligkeit wird aber durch das Zweitglied negiert. In ähnlicher Weise wie bei -frei entwickeln solche Wortbildungsmuster eine gewisse Suggestionskraft, insofern, als schon über die Konstruktion eine mögliche Beeinträchtigung suggeriert wird, deren Wirksamkeit aber gleichzeitig im konkreten Fall negiert wird. Hingegen sind die in gewissem Sinne antonymischen Zweitglieder empfindlich, sensibel nicht spiegelbildlich auf negative Bewertung festgelegt, sondern können genauso gut erwünschte potenzielle Veränderbarkeit ausdrücken. Vgl. auch hier einige Belege:

(46) „Das System ist zu 100 Prozent virensicher“, sagte ein arvato-Sprecher. (gea.de vom 3.9.2005)

(47) Auch die Ost-Kollegen trinken inzwischen Bier mit ihm, alles „PDS-resistente“ Leute und Studierte. (archiv.tagesspiegel.de vom 29.8.2005)

(48) Burggraf sagte, mit dem neuen Spezialfahrzeug könnten Krankentransporte etwa in die Isolierstation künftig absolut keimdicht erfolgen. (fr-aktuell.de vom 10.3.2006) 
(49) Unter „ferner liefen“ rangieren dagegen die dünnen Tablet-PCs, die mit einem Stift über den druckempfindlichen Bildschirm bedient werden können statt über eine Tastatur: Auf sie entfällt weniger als ein Prozent. (handelsblatt.com vom 13.3.2006) ${ }^{14}$

Auch hier ist wieder deutlich eine fachsprachliche Aura erkennbar, die solchen Bildungen anhängt, beliebt ist dieses Muster dann gerade auch in der Werbesprache (wie auch die Privativbildungen).

2.6 Positive/negative Wirkung zeigend: Y hat negative/positive Auswirkung auf X

Zum Schluss soll noch ein in der „Halbsuffix“-Diskussion ebenfalls häufig genanntes antonymisches Zweitglied-Paar aufgeführt werden: freundlich und feindlich, vgl.:

\begin{tabular}{|c|c|c|}
\hline Suffixe & gebundene Zweitglieder & Zweitglieder \\
\hline \multicolumn{3}{|c|}{ Y hat negative Auswirkung auf $\mathrm{X}$} \\
\hline & & feindlich (rückenfeindlich) \\
\hline \multicolumn{3}{|c|}{ Y hat negative Einstellung zu X } \\
\hline & & feindlich (fussballfeindlich) \\
\hline \multicolumn{3}{|c|}{ Y hat positive / keine negative Auswirkung auf $X$} \\
\hline & & $\begin{array}{l}\text { freundlich (umweltfreundlich, } \\
\text { wissenschaftsfreundlich) }\end{array}$ \\
\hline \multicolumn{3}{|c|}{ Y hat positive Einstellung zu X } \\
\hline & & $\begin{array}{l}\text { freundlich (gewerkschafts- } \\
\text { freundlicher Wirtschaftsweiser) }\end{array}$ \\
\hline
\end{tabular}

Tabelle 8: freundlich vs. feindlich

Was in der Literatur häufig als ein typisches Zeichen der Bedeutungsverblassung gesehen wird, ist die Tatsache, dass bei diesen Bildungen auch Bezugssubstantive möglich sind, die nicht als Agens angesehen werden können (wie im viel zitierten Beispiel hautfreundliche Seife). Allerdings zeigt eine (zugegebenermaßen nicht systematische) Durchsicht von Belegen etwa im Portal Wortschatz der Universität Leipzig, dass in den meisten Fällen Bezugssubstantive auftreten, die als Handlungen oder Handlungsresultate von Individuen, Personengruppen oder Institutionen aufgefasst werden können und somit ein Agens durchaus implizit ist, vgl. das forschungsfeindliche Gentechnikgesetz der Grünen; ... weil sich 1529 im Zuge der Reformation eine bilderfeindliche Stimmung durchsetzte; ausländerfreundliche Politik. ${ }^{15}$ Einige weitere Belege:

(50) Soll nun der Rest der Welt dem nordkoreanischen Beispiel folgen, also dafür sorgen, daß naturfeindliche industrielle Aktivitäten insgesamt unterbleiben? (welt.de vom 23.11.2005)

(51) Ich habe ja nichts gegen Fußball, also nicht, dass man mich hier als fußballfeindlich bezeichnet, aber mal ehrlich, so viel am Tag? (ngz-online.de vom 10.5.2006)

(52) Die 16 Teilnehmer des Programms haben sich gegenüber der Provinz verpflichtet, die Ränder ihrer Äcker und Weiden, die an Wald, Hecken und Hohlwegen grenzen, haselmausfreundlich verwildern zu lassen. (an-online.de vom 2.9.2005)

14 Dieser Beleg zeigt deutlich die Schnelllebigkeit der Entwicklungen im Computerbereich.

15 Belege aus dem Portal Wortschatz der Universität Leipzig, http://wortschatz.uni-leipzig.de/, 31.3.2011. Es finden sich nur wenige Belege, bei denen das Bezugssubstantiv nichtbelebt und auch nicht indirekt auf ein Agens beziehbar ist, darunter rückenfeindliches Gefährt, nutzerfeindliche Technik, bürstenfreundliche Glatze und badefreundliche [Temperatur-] Werte. 
(53) Der gewerkschaftsfreundliche Wirtschaftsweise Peter Bofinger ... (welt.de vom 9.9.2006)

Es finden sich sogar einige Belege mit unfreundlich als abstufendem Antonym zu freundlich, vgl. etwa:

(54) ... einen kompetenten Adressaten für ihren Zorn auf viele unsinnige Gesetze, weltfremde Verordnungen oder bürgerunfreundliche Behörden. (abendblatt.de vom 29.5.2005)

(55) „Im Test fiel uns das Angebot durch eine besonders kundenunfreundliche Tarifgestaltung auf", kritisiert Stollberger. (focus.msn.de vom 26.6.2006)

Die grundsätzliche (antonymische) Präfigierungsmöglichkeit auch innerhalb der Wortbildung jedenfalls zeigt die Vitalität des Wortbildungsmusters. Auch dies kann als deutlicher Indikator gegen eine naturgesetzliche Grammatikalisierung hin zum Suffix gelesen werden.

\section{3. $\quad$ Fazit}

Wie geht es den „Halbsuffixen“? Im vorliegenden Beitrag habe ich zu zeigen versucht, dass es dem mit diesem Begriff häufig (auch) bezeichneten Gegenstandsbereich blendend geht - er ist hochproduktiv und scheint in besonderem Maße dem kommunikativen Bedürfnis nach deutlichen, aber schematisierenden klassifikatorischen Abstufungen in bestimmten (häufig technisch-fachlich oder gesellschaftlich-bürokratisch orientierten bzw. inspirierten) Diskursen zu entsprechen. Eine systematischere korpusbasierte Sichtung könnte die hier vorgestellten semantisch-funktionalen Gruppen sicher noch weiter ausdifferenzieren. Was die Kategorie „Halbsuffix/Suffixoid“ angeht, so habe ich zu zeigen versucht, dass sie aus meiner Sicht begrifflich unglücklich gewählt ist, dass sie Zusammenhänge und Differenzierungsmöglichkeiten v.a. in Hinblick auf die ebenfalls hochproduktiven partizipialen Zweitglieder verdeckt und dass ihre Herleitung (,Verblassung der Ausgangsbedeutung; Umkehrung der ursprünglichen Bedeutungsbeziehung") nicht immer zutreffend ist. Der Vorschlag, derartige Zweitglieder als ,lexematische Junktionglieder", die Wortbildungsprodukte als „lexematische Junktionsbildungen“ zu bezeichnen, versucht, das Typische dieser Bildungen - eine auf verschiedene Art hergestellte oder genutzte Relationalität und eine darauf aufbauende reihenbildende, schematisierende, aber semantisch deutliche Beziehung zwischen Erstglied und Bezugssubstantiv - positiv zu fassen und so zu verdeutlichen, dass es sich um einen Kernbereich adjektivischer Wortbildung handelt.

\section{Korpora}

COSMAS II (Neuerwerbungen)

Wortschatz Uni Leipzig

Herder-BYU-Korpus

www.google.de

\section{Literatur}

Altmann, Hans/Kemmerling, Silke (2005): Wortbildung fürs Examen. 2., überarb. Aufl. Göttingen: Vandenhoeck \& Ruprecht. (= Linguistik fürs Examen 2).

Ascoop, Kristin (2005): Affixoidhungrig? Skitba! Status und Gebrauch von Affixoiden im Deutschen und Schwedischen. In: Germanistische Mitteilungen 62, S. 17-28.

Barz, Irmhild (2009): Die Wortbildung. In: Dudenredaktion (Hg.): Duden - Die Grammatik. Mannheim etc.: Dudenverlag. S. 634-762. 
Barz, Irmhild et al. (2007): Wortbildung - praktisch und integrativ. Ein Arbeitsbuch. 4., überarb. Aufl. Frankfurt a.M. u.a.: Lang. (= Leipziger Skripten 2).

canoonet - Deutsche Wörterbücher und Grammatik. (o. J.) URL: http://www.canoo.net/.

Donalies, Elke (2007): Basiswissen Deutsche Wortbildung. Tübingen/Basel: Francke. (= UTB 2876).

Donalies, Elke (2005): Die Wortbildung des Deutschen. Ein Überblick. 2., überarb. Aufl. Tübingen: Narr. (= Studien zur deutschen Sprache 27).

Dudenredaktion (Hg.) (1995): Duden - Die Grammatik. (Duden 4). 5., überarb. Aufl. Mannheim/Leipzig/ Wien/Zürich: Dudenverlag (= Wellmann 1995).

Eichinger, Ludwig M. (1988): Die Wörter, die mein Sohn macht. Natürlichkeit und die Wortbildung des Adjektivs. In: Grazer Linguistische Studien 30, S. 21-36.

Eichinger, Ludwig M. (2000): Deutsche Wortbildung. Eine Einführung. Tübingen: Narr. (= narr Studienbücher).

Eichinger, Ludwig M. (2007): Adjektivische Wortbildung im Deutschen. In: Di Meola, Claudio et al. (Hg.): Perspektiven Zwei. Akten der 2. Tagung Deutsche Sprachwissenschaft in Italien. Rom: Istituto Italiano di Studii Germanici. S. 113-128.

Eisenberg, Peter (2006): Grundriss der deutschen Grammatik 1. Das Wort. 3., durchges. Aufl. Stuttgart/ Weimar: Metzler.

Elsen, Hilke (2009): Affixoide: Nur was benannt wird, kann auch verstanden werden. In: Deutsche Sprache 37, S. 316-333.

Erben, Johannes (2006): Einführung in die deutsche Wortbildungslehre. 5., durchges. u. erg. Aufl. Berlin: Erich Schmidt. (= Grundlagen der Germanistik 17).

Fandrych, Christian (1993): Wortart, Wortbildungsart und kommunikative Funktion. Adjektivische Possessiv- und Privativbildungen im heutigen Deutsch. Tübingen: Niemeyer.

Fleischer, Wolfgang/Barz, Irmhild (2007): Wortbildung der deutschen Gegenwartssprache. 3., unveränd. Aufl. Tübingen: Niemeyer.

Graefe, Karen (2009): Eine korpuslinguistische Untersuchung zur Produktivität des Adjektivsuffixes - $i v$. Leipzig, unveröffentlichte Magisterarbeit.

Kühnhold, Ingeborg/Putzer, Oskar/Wellmann, Hans (1978): Deutsche Wortbildung. Typen und Tendenzen in der Gegenwartssprache. Dritter Hauptteil: Das Adjektiv. Düsseldorf: Schwann.

Latour, Bernd (1976): „,innerparteilich“ - „,parteiintern“. Zur Konkurrenz zweier gegenwartssprachlicher Wortbildungsmuster. In: Deutsche Sprache 4, S. 336-350.

Lohde, Michael (2006): Wortbildung des modernen Deutschen. Ein Lehr- und Übungsbuch. Tübingen: Narr. (= narr Studienbücher).

Motsch, Wolfgang (2004): Deutsche Wortbildung in Grundzügen. 2., überarb. Aufl. Berlin/New York: de Gruyter. (= Schriften des Instituts für Deutsche Sprache 8).

Munske, Horst Haider (2002): Wortbildungswandel. In: Habermann, Mechthild/Müller, Peter O./Munske, Horst Haider (Hg.): Historische Wortbildung des Deutschen. Tübingen: Niemeyer. S. 23-40

Naumann, Bernd (2000): Einführung in die Wortbildungslehre des Deutschen. 3., neubearb. Aufl. Tübingen: Niemeyer. (= Germanistische Arbeitshefte 4).

Schmidt, Günter Dietrich (1987): Das Affixoid. Zur Notwendigkeit und Brauchbarkeit eines beliebten Zwischenbegriffs der Wortbildung. In: Hoppe, Gabriele et al. (Hg.): Deutsche Lehnwortbildung. Tübingen. S. 53-101.

Weinrich, Harald (2003): Textlinguistik der deutschen Sprache. 2. Aufl. Unter Mitarbeit von Maria Thurmair, Eva Breindl und Eva-Maria Willkop. Hildesheim: Olms.

Prof. Dr. Christian Fandrych

Herder-Institut, Philologische Fakultät, Universität Leipzig

Beethovenstr. 15

D-04107 Leipzig

E-Mail: fandrych@uni-leipzig.de 